Giovanni TALLARICO

Université de Vérone

http://dx.doi.org/10.18778/8088-785-5.18

\title{
« Cinquante nuances de board » : anglicismes néologiques et équivalents autochtones dans le domaine des sports de glisse
}

\begin{abstract}
Résumé
Dans notre article nous faisons brièvement état des relations entre sport, terminologie et emprunts dans la dynamique néologique actuelle, en soulignant le poids des anglicismes et le travail d’aménagement mené par la Commission spécialisée de terminologie et de néologie des sports. Notre étude concerne un échantillon d'une petite cinquantaine de termes officiels relevant des sports de glisse, dont nous illustrons les relations sémantiques. Après avoir analysé la présence des emprunts et de leurs équivalents autochtones dans quelques dictionnaires contemporains, généraux et de spécialité, nous mesurons la circulation de ces termes dans la base de données Neoveille, dans le site L'Équipe.fr et dans le web francophone. Il s'avère que les emprunts sont beaucoup plus fréquents que leurs équivalents français, pour des raisons historiques, géographiques et socioculturelles. Pour finir, on peut remarquer que la variation affecte le vocabulaire des sports de glisse à plusieurs niveaux et que l'équivalence sémantique entre termes officiels et emprunts s'avère parfois problématique.
\end{abstract}

Mots-clés : néologie, emprunts, vocabulaire du sport, terminologie, variation, équivalence sémantique. 


\section{Summary}

Our paper starts by providing a brief report on the connections between sports, terminology and loanwords in contemporary French, highlighting the role of anglicisms and the important work carried out by the Commission spécialisée de terminologie et de néologie des sports. Our study deals with a sample of about 50 official terms from boardsports, which we illustrate through the semantic relations that these terms have. After having analysed the presence of loanwords and of their French equivalents in some contemporary dictionaries, both general and specialized, we try to measure the diffusion of these terms in Neoveille database, in L'Équipe.fr website and in Francophone web. It turns out that loanwords are much more widespread than their French equivalents, mainly for historical, geographical and socio-cultural reasons. Finally, we remark that variation affects boardsport vocabulary at more than one level and that semantic equivalence between official terms and loanwords is at times problematic.

Keywords: neology, loanwords, sports vocabulary, terminology, variation, semantic equivalence.

\section{Introduction}

Phénomène global s'il en est, le sport est un terrain d'investigation stimulant pour le lexicologue, car il permet d'observer l'évolution des pratiques à travers leurs dénominations ${ }^{1}$. À l'exclusion de quelques disciplines à caractère surtout local (comme la pelote basque, la pétanque, etc.), les activités sportives sont soumises aux mêmes règles, partagées à l'échelle mondiale, ainsi que le sont les matériels et les équipements utilisés par les pratiquants. En même temps, le vocabulaire sportif se mondialise, et cette mondialisation se fait le plus souvent par l'intermédiaire de l'anglais.

1 Par cette contribution, nous poursuivons la réflexion que nous avons entamée il y a quelques années, avec la collaboration à un dictionnaire du sport conçu et élaboré à l'Université de Vérone sous la direction de Pierluigi Ligas, le Dictionnaire alphabétique et analogique $d u$ français des activités physiques et sportives (DAAFAPS) (2016). La réflexion se poursuit grâce au Laboratoire de lexicographie sportive annexe (www.univr-lls.net) (consulté le 15/02/2018). 
Le langage des sports se caractérise par une vitalité remarquable, qui répond en premier lieu à des besoins de dénomination ${ }^{2}$. Georges-Ray Jabalot, ancien Président de la Commission spécialisée de terminologie et de néologie du sport, explique les raisons de ce foisonnement : « Aujourd'hui, non seulement de nouvelles 'disciplines sportives' apparaissent de façon continuelle, mais encore, au sein des disciplines officielles existantes, se développent de nouvelles formes de pratiques échappant aux fédérations sportives censées les encadrer» (Jabalot, 2012: 18).

Traditionnellement, les apports au lexique sportif sont pour une grande partie de matrice externe, notamment anglo-américaine ${ }^{3}$. Comme le précise Sablayrolles,

L'introduction ou l'amélioration d'activités physiques pratiquées dans d'autres pays ont conduit le français à importer des mots - noms, verbes ou adjectifs essentiellement - pour le nom de ces sports bien sûr, mais aussi pour les principales actions relatives à leur pratique, leurs acteurs, les qualités ou défauts qu'on y observe, le matériel utilisé, en particulier les vêtements, etc. (Sablayrolles, $2005: 41)$.

La forte médiatisation du vocabulaire sportif et la « porosité » de ses frontières lexicales font la spécificité de ce domaine langagier. Anna Giaufret en résume efficacement les enjeux :

S'il est légitime de s'interroger sur la nature du discours sportif, sur l'existence d'une ou de plusieurs langues de spécialité des sports, qui seraient plutôt des sous-ensembles de la langue générale, maîtrisés par des communautés de praticiens et de passionnés, il est bien évident qu'il existe une terminologie spécifique des différentes disciplines sportives, celles-ci ayant besoin de nommer les équipements, les techniques, les espaces qu’elles utilisent (Giaufret, 2012 : 57).

La place centrale du sport dans la société et dans les médias est à l'origine d'une activité terminologique de plus en plus intense. On peut affirmer à juste titre que «l'importance que le sport et les terminologies du sport ont prise depuis quelques années fait de ce domaine un secteur d'activités de dimension mondiale » (Depecker, 2012a : 11-12). La présence de nombreux glossaires, lexiques et banques de données à caractère sportif témoigne de ce regain d'inté-

2 «De nouvelles pratiques et modalités de pratique génèrent des vocabulaires nouveaux » (Tetet, 2000a : 503).

3 Cf. Gritti (1985); Depecker (1995) ; Bernard-Béziade (2010) ; Tallarico (2014). 
rêt. Par exemple, dans FranceTerme 4 figurent aujourd'hui 331 recommandations officielles pour les sports. C'est un chiffre qui ne cesse d'augmenter : en 2009, il n'y en avait que $81^{5}$.

La reconnaissance institutionnelle de ce secteur remonte à 1984, lorsque fut créée la première Commission ministérielle de terminologie du sport (aujourd'hui, Collège d'experts). Rattachée au Ministère de la Ville, de la Jeunesse et des Sports, elle a pour tâche principale de proposer des équivalents français pour les anglicismes du domaine.

\section{La terminologie des sports de glisse}

Dans le cadre de cet article, nous avons choisi de nous pencher sur le domaine des sports de glisse, en raison de sa prolifération importante au cours des dernières années ${ }^{6}$ et du taux élevé d'emprunts qui le caractérise ${ }^{7}$. Tetet apporte des compléments culturels intéressants à propos de cette " macro-discipline » :

Les sports de glisse nés en Californie dans les années 50, d'où l'appellation de sports californiens donnée par les sociologues, se développent en France dans les années 60-70. Ils sont d'abord le fait de jeunes adeptes qui recherchent le plaisir des sensations fortes dans la maîtrise des éléments naturels, eau et air, à l'aide d'appareillages nouveaux (Tetet, 2000a : 519).

Comment les sports de glisse sont-ils définis dans les dictionnaires généraux ? Si Le Petit Robert 2017 propose une simple énumération (« Sports de glisse: ensemble des sports où l'on glisse (ski, snowboard, surf, planche à voile, barefoot, bodyboard, halfpipe, kitesurf, roller, skate-board, skeleton, bobsleigh...)») ${ }^{8}$, Le Petit Larousse 2016 opte pour une répartition en typologies : «Sports de glisse: Ensemble des sports où l'on glisse sur la neige, la glace ou l'eau (ski, patinage, surf, etc.) ou que l'on pratique sur roulettes (skateboard, roller, trottinette, etc.), voire sur roues (bicross)».

4 Le site au travers duquel le public peut avoir accès aux termes parus au Journal Officiel (www.culture.fr/franceterme) (consulté le 15/02/2018).

5 Cf. Brancaglion (2009).

6 Cf. Attali, Saint Martin (éds.) (2010 : 59-61).

7 Cf. Sablayrolles (2005) ; Monrozier (2016).

8 La définition du dictionnaire Hachette 2016 est pratiquement la même, avec quelques exemples en moins. 
Le Journal Officiel, pour sa part, propose une définition de «sports de planche » et non pas de « sports de glisse», la conversion verbe-nom (glisser $\rightarrow$ glisse) étant sans doute jugée comme impropre : «Sport consistant à évoluer sur l'eau, la neige, le sol ou dans l'air, en équilibre sur un engin, appelé communément planche, conçu à cet effet ».

Signalons aussi qu'une enquête de l'INSEE/Jeunesse et sports ${ }^{9}$ répartit les sports de glisse en quatre catégories : le ski, la glisse urbaine (roller et skate), la glisse nature (planche à voile, surf, bodyboard, surf des neiges) et la glisse sur glace (patinage).

\subsection{Corpus}

À partir de FranceTerme, nous avons retenu 48 termes officiels relevant des sports de glisse, dont 43 ont trait aux disciplines et cinq désignent les noms de pratiquants.

\section{Disciplines}

char à cerf volant

glisse aérotractée

paret

patin en ligne

planche à bras, planche acrobatique de neige, planche acrobatique terrestre, planche à cru, planche aérienne, planche à deux roues, planche à genoux, planche à rame, planche à roues, planche à roulettes, planche à roulettes en ligne, planche artistique de neige, planche à voile, planche à voile terrestre, planche d'eau vive, planche de descente, planche de dune, planche de neige, planche de neige tractée, planche de piste, planche de plage, planche de rue, planche de sillage, planche de sport, planche de surf, planche hors-piste, planche magnétique, planche nautique, planche serpent, planche terrestre, planche terrestre aérotractée, planche tout-terrain, planche ventrale, planche volante, planche-kayak

rampe de neige

ski-parapente, ski-selle, ski volant

\section{Pratiquants \\ aéroplanchiste \\ aquaplanchiste}

9 Citée dans Garel (2012). 
nivoplanchiste

planchiste

véliplanchiste.

\subsection{Morphologie}

Il est à noter que 35 parmi ces termes sont des synapsies ${ }^{10}$ (planche à bras, planche de dune, planche de sillage, etc.) ou des unités terminologiques (comme planche acrobatique de neige) où le mot planche figure comme «tête ", en lieu et place des composés anglais avec board (paddleboard, sandboard, wakeboard, etc.). Les joncteurs prépositionnels les plus exploités dans cette « série analogique » sont $\grave{a}$, qui indique le moyen (planche à genoux, planche à bras, etc.) et $d e$, qui situe le lieu de pratique et l'objectif (planche de rue, planche de descente, etc.). Dans la liste figurent également huit mots composés, dont quatre composés hybrides parmi les noms de pratiquants (aéroplanchiste, aquaplanchiste, nivoplanchiste ${ }^{11}$, véliplanchiste), un mot dérivé (planchiste) et un mot simple, paret. Nous n'entrerons pas dans le détail de la formation des équivalents autochtones, mais d'après le modèle de Sablayrolles (2016) on peut y relever d'emblée plusieurs calques morphologiques (comme patins en ligne pour in line skate) et quelques synthèses néologiques (ou "trous comblés»), comme planche d'eau vive pour hydrospeed.

\subsection{Système conceptuel}

Depecker fait état de la difficulté que la Commission spécialisée de terminologie et de néologie a rencontrée dans le traitement des

concepts des planches utilisés dans le sport. Ces planches sont de toutes sortes, elles sont utilisées dans de multiples environnements, et se comptent par dizaines de types différents [...]. Il a fallu, pour s'y retrouver, procéder comme dans tout travail terminologique : identifier les objets, relier les concepts entre eux, les classer à leur rang et y ajuster les termes anglais et français correspon dants. (Depecker, 2012a : 12-13).

10 Unités de signification composées de plusieurs morphèmes lexicaux, d'après la terminologie de Benveniste, reprise par Sablayrolles.

11 Qui exploite le formant nivo-, productif en géographie et en géophysique (nivoglaciaire, nivologie, etc.) 
En suivant cette même approche, nous avons essayé de reconstituer le réseau conceptuel des planches de sport (l'hyperonyme « macro ", super-ordonné par rapport à tous les autres éléments), en identifiant des relations logiques de type générique, fondées sur l'hyperonymie et l'hyponymie, et des relations de coordination ou, pour mieux dire, de co-hyponymie.

Nous présentons ci-dessous le tableau 1 consacréà la glisse urbaine, où figure en haut l'hyperonyme planche terrestre avec ses quatre hyponymes; pour l'un d'eux, planche à roulettes, il existe cinq termes « sous-ordonnés », dont planche de rue qui n'indique pas le matériel, mais uniquement la pratique correspondante.

Tableau 1. Schéma de la glisse urbaine

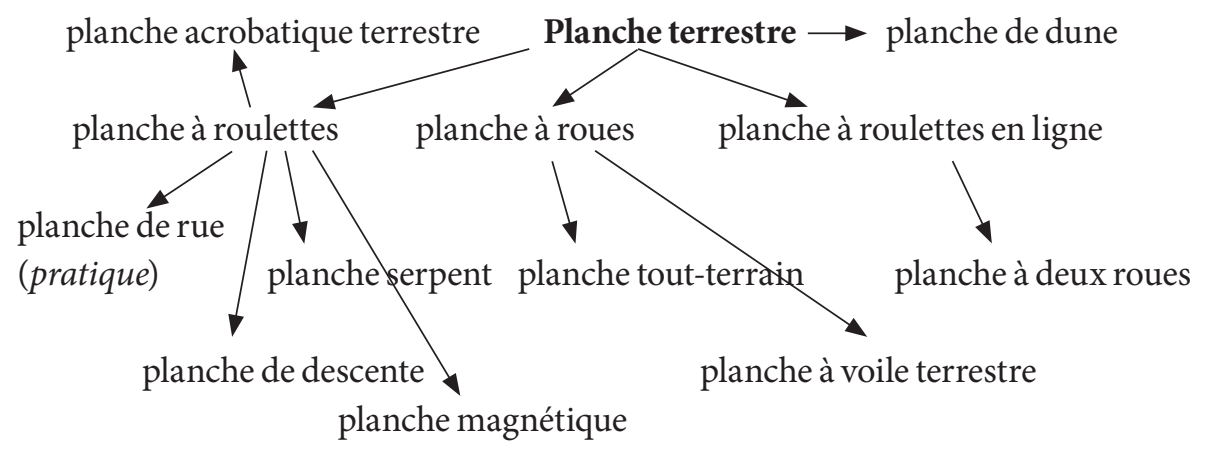

Dans la catégorie "glisse aquatique » nous avons relevé 12 termes au total, dont 11 hyponymes directs de planche nautique. Quelques-uns, comme planche-kayak, indiquent à la fois l'engin et la pratique (voir tableau 2).

Tableau 2. Schéma de la glisse aquatique

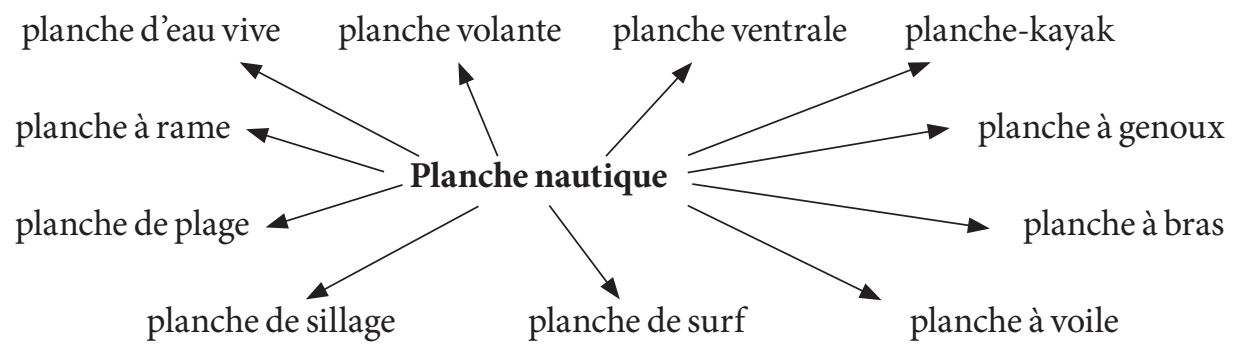


Pour finir, les planches de neige s'articulent en quatre typologies et donnent vie à deux pratiques spécifiques, la rampe de neige (halfpipe) et la planche artistique de neige (ou snowpark boarding) (voir tableau 3).

Tableau 3. Schéma de la glisse sur neige

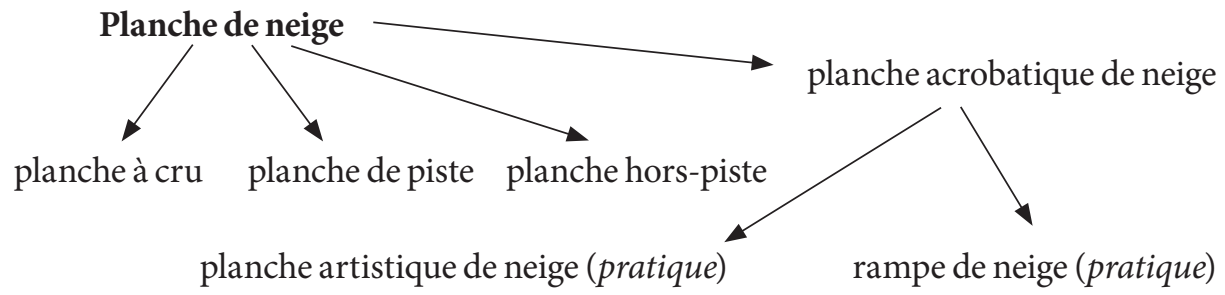

\section{Les emprunts dans les sports de glisse et leurs équivalents en lexicographie}

La lexicographisation, à savoir la présence d'un mot dans les dictionnaires, est sans aucun doute un indice important de lexicalisation. Il s'agit maintenant d'évaluer la présence des emprunts dans le domaine des sports de glisse et de leurs équivalents autochtones dans quelques répertoires lexicographiques actuels.

Notre « méta-corpus » se compose du Petit Robert 201712, du Petit Larousse illustré $2016{ }^{13}$, du Dictionnaire Hachette $2016^{4}$ et du Dictionnaire alphabétique et analogique du français des activités physiques et sportives (DAAFAPS) ${ }^{15}$. Nous avons préféré ce dernier au Robert des Sports (Petiot, 1982), qui a une nomenclature assez réduite (environ 5000 entrées ${ }^{16}$ ) et ne tient pas compte des évolutions récentes, alors que le DAAFAPS possède environ 17000 entrées et reflète l'usage contemporain.

\footnotetext{
12 Dorénavant PR17.

13 Dorénavant PL16.

14 Dorénavant DH16.

15 Cf. infra, note 1. Pour une présentation de ce dictionnaire, voir Ligas (2013).

16 Tetet (2000b) a calculé 5250 entrées.
} 
Commençons par les termes liés aux disciplines. Si l'on prend en compte les officialismes, dans $P R 17$ figurent 7 termes sur 4317 , souvent à l'entrée même de l'équivalent étranger. $P L 16$ et $D H 16$, pour leur part, ne sont pas très accueillants (ou sinon très descriptifs de l'usage) à l'égard des termes officiels : seuls deux et trois termes y sont présents respectivement, notamment planche à roulettes et planche à voile pour les deux dictionnaires, auxquels DH16 ajoute patin en ligne. Dans le DAAFAPS, en revanche, figurent 10 termes officiels sur 43, dont char à cerf volant, glisse aérotractée et planche de dune, qui ne sont répertoriés dans aucun autre dictionnaire du corpus.

En ce qui concerne les équivalents étrangers, le même nombre (sept) figure dans PR17 et PL16:

PR17 : bodyboard, kite(surf), skateboard, snowpark, snowboard, speed-sail, windsurf

PL16: Bodyboard, kite(surf), half-pipe, skateboard, skysurfing, snowboard, Windsurf

Le traitement de $\mathrm{DH} 16$ est plus extensif, avec 13 équivalents étrangers figurant dans la nomenclature : bodyboard, freeride, halfpipe, hydrospeed, kite $(\text { surf })^{18}$, skateboard, skatepark, skysurf(ing), snowboard, snowpark, speedsail, wakeboard et windsurf.

Il n'est pas surprenant de constater qu'un dictionnaire de spécialité comme le DAAFAPS est de loin le plus complet, avec 25 équivalents (dont kneeboard, paddleboard, sandboard, etc.) pour les activités correspondant aux termes officiels, dont les définitions sont souvent tirées du Journal Officiel.

Pour ce qui est des noms de pratiquants, planchiste (attesté depuis 1978 pour $P R 17)$ est le terme générique officiel, désignant tout pratiquant de sports de planche. S'ensuivent naturellement d'autres dénominations ayant recours à des formants savants, qui donnent lieu à des composés hybrides :

aéroplanchiste (skyboarder, pratiquant de planche aérienne) aquaplanchiste (boardsurfer, pratiquant de planche nautique) nivoplanchiste (snowboarder, pratiquant de planche de neige) véliplanchiste (windsurfer, pratiquant de planche à voile).

17 Notamment patin en ligne, planche à roulettes, planche à voile, planche de neige, planche de surf, planche ventrale et planche volante.

18 La variante kiteboard est aussi mentionnée. 
Parmi ces termes, seulement planchiste et véliplanchiste sont recensés dans notre corpus lexicographique, mais à l'unanimité ; le premier, d'ailleurs, est interprété comme un synonyme du second. Aucun équivalent étranger n'est répertorié dans les dictionnaires pris comme corpus d'étude, sauf l'emprunt francisé snowboardeur $(D H 16)^{19}$.

À la suite du traitement lexicographique que nous venons d'illustrer, notamment celui de DH16, il convient de mitiger les propos de Tetet, selon laquelle le sport est « une réalité que le lexicographe de dictionnaires généraux maîtrise mal, préférant faire porter son attention sur d'autres domaines » (2000b : 199). Du moins pour le domaine des sports de glisse, les dictionnaires se montrent plus accueillants et attentifs aux évolutions lexicales que dans le passé.

\section{Circulation des termes et de leurs équivalents étrangers}

Il s'agit maintenant de mesurer la circulation des termes officiels et de leurs équivalents dans un corpus web comprenant:

1. Neoveille ${ }^{20}$, une « plateforme multilingue de veille et de suivi des néologismes à partir de corpus contemporains de très grande taille dans sept langues $»^{21}$. C'est un outil qui permet d'obtenir des résultats avec peu de «bruit » et aussi, par sérendipité, de trouver ce que l'utilisateur ne cherchait pas (comme par exemple des dérivés), juste en tapant la base du mot ;

2. L'Équipe.fr, le site du quotidien français de référence pour le sport;

3. Google, limité aux pages en français. Une mise en garde est nécessaire sur le « web as corpus». Comme chacun sait, la fiabilité des résultats obtenus grâce à un moteur de recherche généraliste est loin d'être satisfaisante : homonymes, noms de marque ou de produit, affichage de pages dans des langues autres que le français, impossibilité d’avoir

19 Pour ce dérivé, cependant, il pourrait tout aussi bien s'agir d'« une création lexicale réalisée en français à partir d'une racine empruntée " (Sablayrolles $2016: 30$ ).

20 Pour une présentation du projet et de sa structure, nous renvoyons à Cartier (2016).

21 Descriptif disponible à l'adresse www.neoveille.org. 
des résultats uniquement pour la forme demandée 22 , comptages imprécis $^{23}$, etc., voici autant d'aléas pour le chercheur ${ }^{24}$. Nous nous sommes donc contenté de dégager quelques tendances majeures et des écarts flagrants entre les termes examinés : d'un côté les officialismes, de l'autre les équivalents étrangers.

D'après nos recherches, trois cas de figure peuvent se manifester :

1) termes avec de faibles attestations (grosso modo moins de 1000 résultats sur Google), pour lesquels il est malaisé d'avancer des hypothèses ;

2) cas où le terme français est plus répandu que l'emprunt ;

3) cas où l'emprunt est plus répandu que le terme autochtone (la grande majorité).

De manière générale, l'absence ou l'extrême rareté d'attestations dans L'Équipe indiquent que la pratique de ces disciplines dans les pays de langue française est encore très limitée, comme pour planche terrestre aérotractée (en anglais kite skateboarding). Si l'hyperonyme, rare dans le corpus web, glisse aérotractée (kite sports) répond à une nécessité de conceptualisation, plutôt que de communication réelle, le binôme planche acrobatique de neige/snowpark board est lui aussi peu représenté pour les mêmes raisons.

Les cas où le terme français est plus répandu que l'emprunt ne sont que trois: les dénominations les plus " anciennes " planche à roulettes, planche à voile et planche de surf se portent encore très bien et concurrencent, voire dépassent en nombre d'attestations, les équivalents étrangers skateboard, windsurf et surfboard.

Venons-en maintenant aux cas où l'emprunt circule mieux, voire supplante ${ }^{25}$ l'équivalent autochtone. Il s'agit, et de loin, de la catégorie la mieux représentée, avec 32 cas sur 43. Par exemple, pour les binômes planche à bras/paddleboard, planche à cru/snowskate, planche à deux roues/waveboard, etc., le rapport entre terme officiel et emprunt dans Google est au moins d'1 à 1000, sans mentionner naturellement des emprunts lexicalisés tels que snowboard, bodyboard ou kitesurf.

22 Par exemple, une recherche sur la forme freeriding affiche aussi les résultats pour freeride.

23 Dans notre expérience, assez souvent les résultats affichés sont de l’ordre des milliers, puis, quand on fait défiler les pages, ils se limitent à quelques dizaines seulement.

24 Cf. Sablayrolles (2009).

25 Pour Depecker, la supplantation se produit lorsqu' « un terme étranger tend à prendre la place d'un terme usuel dans une langue, au point de le remplacer » (Depecker 2012b : 25). 
Avec un brin d'optimisme, Garel observe que

la brièveté des termes empruntés est fréquente et attrayante, mais elle ne suffit pas à expliquer leur prééminence croissante : 'stand up paddle surf', qui désigne l'ensemble constitué d'une planche nautique et d'une rame [...] n'est pas encore supplanté par 'planche à rame', pourtant plus concis. (Garel, 2012 : 33)

Dans ce cas aussi, l'écart quantitatif entre la forme anglo-américaine stand up paddle surf 26 et l'équivalent français planche à rame (un rapport de 1500 à 1 dans Google) est si net qu'il nous paraît peu probable que la donne puisse changer rapidement.

Cette remarque nous amène à nous pencher sur les motivations pouvant expliquer la grande diffusion des emprunts dans le domaine des sports de glisse. Tout d'abord, il peut être instructif de constater que l'origine de beaucoup de disciplines est américaine, océanienne ou, de toute façon, de matrice anglo-saxonne. Il s'agit de pratiques assez, voire très répandues en France aussi, comme le mountainboard (ou planche tout-terrain), le skateboard, le waveboard et le windsurf, qui sont toutes nées aux États-Unis. Plus particulièrement, à Hawaï apparaissent le bodyboard, le stand up paddle et le surfboarding. L'Océanie est le berceau de disciplines comme le kite-buggy (ou char à cerf-volant), le paddleboard et le wave-ski (ou planche-kayak). Enfin, le streetboard ou snakeboard (ou planche serpent) est originaire d'Afrique du Sud.

Toutefois, l'anglais s'impose aussi pour les dénominations de pratiques qui sont originaires de France, comme le bladeboard, le kitesurf, le sky-surf, le speed riding et le speed sail, ce qui en dit long sur le rôle de cet idiome comme lingua franca pour les sports de glisse. On comprendra la difficulté de la Commission de terminologie, qui s'est trouvée confrontée à des concepts nés en France mais dont l'acte de baptême s'est fait directement en anglais !

\section{Valeurs et fonctions de l'emprunt}

Comment expliquer cette vague d'emprunts dans le domaine des sports de glisse? Plusieurs raisons concourent à cette « anglomanie», notamment des raisons historiques, qui font que le choix de l'anglais dans le sport est souvent perçu

26 Un article récent du quotidien La Croix confirme la vague de cette discipline : http://www.la-croix.com/Journal/En-France-stand-paddle-glisse-plus-plusvite-2016-10-28-1100799543 (consulté le 15/02/2018). 
comme « non marqué » 27 , et des raisons géographiques (on parle justement de «sports californiens »). Mais il ne faut pas sous-estimer l'aspect socio-culturel, qui semble parfois décisif: parmi beaucoup d'autres linguistes, Rousseau (2005) souligne les motivations d'ordre identitaire et symbolique pouvant expliquer les difficultés d'implantation des termes nouveaux destinés à remplacer les emprunts dans un milieu professionnel.

Dans la même veine, Garel évoque un facteur qui a tout son poids dans la circulation des emprunts dans les blogs, forums, publications et sites spécialisés :

Faire appel à l'anglais a sans doute aussi pour intérêt de donner parfois à ce dont on parle, et donc au locuteur, un aspect valorisant [...]. Ajoutons que l'emprunt à une langue étrangère peut marquer le sentiment d'appartenance à une communauté, davantage que le souci de se faire comprendre au-delà de son groupe de pratiquants. (Garel, $2012: 33$ )

La dimension culturelle et identitaire du vocabulaire des sports de glisse, qui fait la part belle aux emprunts, est aussi mise en valeur par Monrozier :

Ces sports ont comme point commun une philosophie de liberté et d'indépendance qui leur fait éviter toute forme de structure et organisation. Dès lors, les recommandations terminologiques n'ont aucune prise sur cet univers. Autre aspect fondamental de l'état d'esprit propre à ces sports : la décontraction, qui semble devoir s'exprimer prioritairement en anglais [...] Ajoutons à cela l'esprit de communauté de la glisse, porteuse d'une identité forte. Celle-ci s'exprime dans un certain nombre d'habitudes partagées : l'emploi d'un langage spécifique, opaque au monde extérieur, en fait partie, au même titre que la tenue vestimentaire ou la musique. (Monrozier, $2016: 69-70$ )

Il faut aussi signaler que les deux corpus analysés, métalexicographique et web, témoignent d'une présence significative d'apocopes telles que kite, skate, snow et wake, en lieu et place des emprunts kitesurf, skateboard, snowboard et wakeboard. Ce phénomène indique sans doute la volonté des locuteurs de marquer leur appartenance à un milieu; de toute évidence, l'emploi du seul terme autochtone planche ne pourrait pas remplir cette fonction. Voici sans doute un autre obstacle à l'implantation des propositions terminologiques ${ }^{28}$.

27 Cf. note 3 pour quelques références bibliographiques.

28 Nous remercions Myriam Paquet-Gauthier pour cette hypothèse, qui reste à creuser. 


\subsection{Phénomènes variationnels}

Le domaine du sport ne fait pas exception : la terminologie en discours est sujette à de fortes variations, dont on trouve la trace dans les dictionnaires et, de plus encore, chez les locuteurs. En ce qui concerne l'orthographe, par exemple, Bodyboard et Windsurffigurent avec initiale majuscule dans PL16 et y sont marqués comme "noms déposés », contrairement à $P R 16$ qui les traite comme des noms communs; $D H 16$ opte pour une solution intermédiaire : la mention «nom déposé » et l'initiale minuscule. De même, dans notre corpus web l'équivalent étranger Rollerblade ('patin en ligne') figure très souvent comme un nom de marque ; les dictionnaires analysés, pour leur part, répertorient tous la forme roller, qui est plutôt l'apocope de rollerskate ('patin à roulettes').

Pour ce qui est de la synonymie, des variations significatives existent pour plusieurs disciplines, un trait qui confirme le caractère néologique de ce vocabulaire. D’après notre corpus web :

- la forme kayak-surf est beaucoup plus fréquente que planche-kayak (qui est le terme officiel);

- la forme aéroplanchiste est plus souvent synonyme de kitesurfeur que de skyboarder 'pratiquant de planche aérienne', comme le voudrait le Journal Officiel;

- downhill longboard est souvent abrégé en downhill ou longboard ou inversé en longboard downhill ;

- paddleboard s'abrège en paddle ou se métamorphose en surf-paddle (d'après Neoveille);

- des équivalents spontanés pour planche de dune sont surf des sables et, de manière locale, snowboard berbère.

En ce qui concerne l'axe diatopique, signalons que, d'après Wikipédia, rampe de neige est un terme employé au Québec; de même, les formes planche à neige (PL16 et DH16) ou planche à snow sont des synonymes québécois du terme officiel planche de neige ${ }^{29}$.

Enfin, en diachronie courte : pour le terme kite, PL16 précise « on dit aussi flysurf ", bien que ce dernier se réfère plutôt à l'ancêtre de cette discipline. Selon Tetet (2000a : 519) l'appellation snowboard a remplacé celle de surf des neiges ${ }^{30}$

29 Cf. Monrozier (2016: 71).

30 Plus récemment, Monrozier confirme que « le terme 'surf des neiges' [...] a disparu au profit de 'snowboard'» $(2016: 71)$. 
(introduite en France à la fin des années $70^{31}$ ) depuis le début des années 90 ; signalons toutefois que, d'après $P R 17$, le terme surf des neiges s'est spécifié et renvoie aujourd'hui à la "glisse pratiquée sur la poudreuse».

\subsection{Sémantique}

Pour ce qui est des relations sémantiques entre emprunts et équivalents autochtones, il faut souligner tout d'abord un risque de confusion référentielle avec le terme officiel planche volante (J.O. 26/11/08), qui dans le discours désigne autre chose qu'un kitesurf: d'après notre corpus, c'est plutôt un synonyme de flyboard (jet-ski) ou de hoverboard, une planche à roulettes permettant de planer au-dessus du sol grâce au magnétisme (comme dans le film Retour vers le futur) ou, dans un sens plus récent, « un véhicule auto stabilisé sur deux roues, doté de deux plateformes indépendantes sous les pieds, pivotant l'une par rapport à l'autre sur l'axe de l'essieu »32.

D’après la Commission spécialisée de terminologie et de néologie du sport, le wave ski «s'inscrit moins dans le domaine du ski que dans celui des planches nautiques » (Garel, $2012: 3$ ). Conciliant la forme d'une planche de surf et celle d'un kayak à siège creux, il permet en effet d'évoluer sur une vague déferlante en étant assis et en s'aidant d'une pagaie double. Voilà pourquoi, selon la Commission, « il est [...] plus juste de désigner cet engin, et la pratique consistant à l'utiliser, par 'planche-kayak' ou 'kayak-surf'" (Garel, 2012:34). Aussi, les efforts d'aménagement linguistique peuvent entraîner une reconceptualisation des termes.

\section{Conclusion}

La néologie officielle dans le domaine des sports de glisse encourage, comme il se doit, l'exploitation des processus traditionnels de formation des mots, tout en essayant de réduire l'impact des anglicismes en français. Mais le chemin paraît encore long. En raison de sa médiatisation et de sa vaste circulation dans

31 Le terme figure encore dans PL16 et dans DH16 (mais ce dernier retient seulement la forme surf de neige).

32 Cf. https://store.urban360.com/definition-micro-mobilite-electrique/ (consulté le 15/02/2018). 
la société, le vocabulaire des sports est particulièrement influencé par la mondialisation, qui se manifeste par le biais des emprunts à l'anglo-américain.

Les difficultés d'implantation terminologiques peuvent également s'expliquer par l'écart entre la prolifération rapide des nouvelles pratiques et le rythme plus graduel du dispositif institutionnel d'enrichissement de la langue française. Ainsi que nous l'apprend le cas de mercatique, proposé dans les années 70 comme équivalent de marketing à une époque où l'anglicisme s'était déjà affirmé, la réactivité est souvent un facteur décisif pour que les termes autochtones s'implantent avec succès dans la langue. À ces facteurs s'ajoutent ceux évoqués par John Humbley, et qui ne semblent pas avoir été suffisamment pris en compte dans les propositions d'équivalents autochtones pour les sports de glisse :

les termes officiels qui ont le plus de chances de s'implanter sont ceux qui conservent une parenté morphologique avec le terme étranger (ex. conteneur pour container), ceux qui favorisent la compréhension (bilan de santé pour check-up), et ceux qui permettent d'éviter des mots dont la prononciation peut poser des problèmes (ingénierie pour engineering) (Humbley, 2000 : 328).

Des pistes de recherche ultérieures se profilent : par exemple, il serait fructueux de se pencher sur les dérivés des noms de disciplines, qui indiquent essentiellement les pratiquants ${ }^{33}$. À ce propos, dans Neoveille nous avons relevé des formes concurrentes telles que kiteur/kitesurfeur, longboardiste/longboardeur et wakeboardeur/wakeboarder. Il serait aussi instructif de suivre l'évolution de nouveaux sports de glisse, dont le wakesurf (qui peut aussi compter sur une association française et une page Wikipédia), le dronesurfing, le handisurf et le no-board.

En conclusion, les sports de glisse risqueraient-ils d'être victimes de ce qu'on appelle la "perte de domaine », notion qui se réfère à « un domaine de l'activité, pour lequel manquent des termes autochtones, forçant la langue à l'emprunt massif » (Depecker, 2012b : 26) ? Il est sans doute encore trop tôt pour l'affirmer et il faudrait en analyser plus en profondeur les discours, mais si l'on relit ce qu'écrivait en début de millénaire Christiane Tetet, à savoir que « les américanismes sont concurrencés, voire remplacés, par des traductions françaises, ou des francisations, à partir du moment où l'activité touche un public d'adultes, connaît une forte extension et lorsque l'instrument ou l'engin support de l'activité est fabriqué en France » (Tetet, 2000a: 522), comme pour beach volley/volley

33 Dans DH16, par exemple, figurent les formes concurrentes skateboardeur et skateur, ainsi que kitesurfeur, rolleur, skysurfeur, snowboardeur et surfeur. 
de plage, windsurf/planche à voile, mountain bike/VTT, etc.; on est bien tenté de dire que cette conclusion est loin de s'appliquer aux dénominations des sports de glisse en français contemporain.

\section{Références bibliographiques}

Attali Michaël, Saint Martin Jean (éds.), 2010, Dictionnaire culturel du sport, Paris, Armand Colin.

Bernard-Béziade Mélanie, 2010, Les anglicismes dans le sport en langue française : modes d'intégration et aspects sociolinguistiques, Berlin, Éditions universitaires européennes.

Brancaglion Cristina, 2009, "Anglicismi nel francese dello sport», in Il linguaggio dello sport, la comunicazione e la scuola, Beatriz Hernán-Gómez Prieto (éd.), Milan, Led, p. 29-42.

Cartier Emmanuel, 2016, « Neoveille, système de repérage et de suivi des néologismes en sept langues », Neologica 10, p. 101-131.

Depecker Loïc, 2012a, "Introduction », in Le langage des sports : identité et typologie, Paris, Société française de terminologie, p. 11-13.

Depecker Loïc, 2012b, « De quelques évènements qui ont déterminé notre souci terminologique dans le sport ", in Le langage des sports : identité et typologie, Paris, Société française de terminologie, p. 23-27.

Depecker Loïc, 1995, "La langue des sports et des loisirs en plein air », in Histoire de la langue française 1914-1945, Gérald Antoine, Robert Martin (éds.), Paris, CNRS éditions, p. 463-505.

Dictionnaire Hachette 2016, 2015, Paris, Hachette.

Garel Jean-Pierre, 2012, "Introduction aux questions d'ordre terminologique dans le domaine du sport ", in Le langage des sports : identité et typologie, Paris, Société française de terminologie, p. 29-39.

Giaufret Anna, 2012, "La terminologie du ski de fond : glossaires, dictionnaires, répertoires ", Études de linguistique appliquée 165, 'Le langage du sport', p. 57-78.

Gritti Jules, 1985, «Le vocabulaire des sports, les anglicismes », in Histoire de la langue française 1880-1914, Gérald Antoine, Robert Martin (éds.), Paris, CNRS éditions, p. 175-191.

Humbley John, 2000, « La terminologie », in Histoire de la langue française 1945-2000, Gérald Antoine, Bernard Cerquiglini (éds.), Paris, CNRS Éditions, p. 315-334. 
Jabalot Georges-Ray, 2012, «Définition et typologie du sport : une question d'actualité ", in Le langage des sports : identité et typologie, Paris, Société française de terminologie, p. 15-21.

Le Petit Larousse illustré 2016, 2015, Paris, Larousse.

Le Petit Robert de la langue française 2017, 2016, Paris, Le Robert.

Ligas Pierluigi, 2013, «Entre langue courante et langue de spécialité : le langage du sport et le Daafaps ", in Dictionnaires, technolectes et terminologie, Leila Messaoudi et al. (éds.), Kénitra, Laboratoire Langage et société, CNRSTURAC, p. 317-334.

Ligas Pierluigi (éd.), 2016, Dictionnaire alphabétique et analogique du français des activités physiques et sportives (DAAFAPS), Vérone, Qui Edit, 2 vol. (A-G, H-Z, $1005+979$ pages).

Monrozier Élisabeth, 2016, « Les anglicismes dans les sports de glisse », Traduire 234, p. 69-73.

Petiot Georges, 1982, Le Robert des sports. Dictionnaire de la langue des sports, Paris, Le Robert.

Rousseau Louis-Jean, 2005, "Terminologie et aménagement des langues », Langages 157, p. 94-103.

Sablayrolles Jean-François, 2016, «Emprunts et influences d'autres langues », in Emprunts néologiques et équivalents autochtones en français, polonais et tchèque, Zuzana Hildenbrand, Alicja Kacprzak, Jean-François Sablayrolles (éds.), Limoges, Lambert-Lucas, coll. « La Lexicothèque », p. 23-35.

Sablayrolles Jean-François, 2009, « ¿ Neologismo o no ? ensayo de clarificación de algunos problemas de incorporación », Revista de investigación lingüística 12, p. 101-122.

Sablayrolles Jean-François, 2005, « Mots du sport, emprunts et langue courante ", in Écrire le sport, Philippe Baudorre, Myriam Boucharenc, Michel Brousse (éds.), Bordeaux, Presses Universitaires de Bordeaux, p. 39-51.

Tallarico Giovanni, 2014, «Traitement de la néologie externe dans la lexicographie sportive : les emprunts dans le DAAFAPS», Neologica 8, p. 63-79.

Tetet Christiane, 2000a, "Le vocabulaire des sports et des loisirs sportifs », in Histoire de la langue française 1945-2000, Gérald Antoine, Bernard Cerquiglini (éds.), Paris, CNRS Éditions, p. 503-526.

Tetet Christiane, 2000b, « Note de lexicographie sportive. Sport et ses dérivés dans la lexicographie française des XIX $\mathrm{X}^{\mathrm{e}}$ et $\mathrm{XX}^{\mathrm{e}} \mathrm{s}$. », Cahiers de lexicologie 77-2, p. 189-200. 